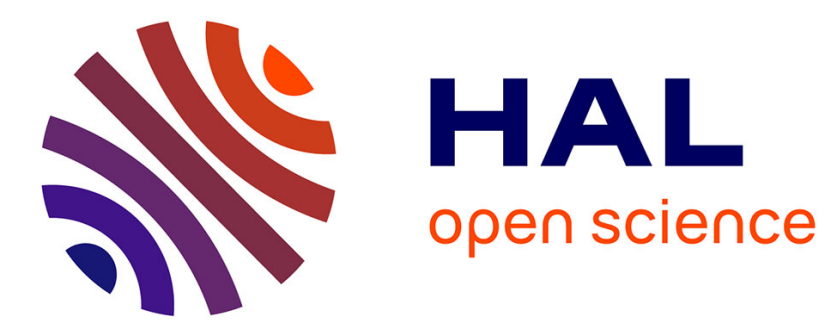

\title{
Interlaminar stress analysis with a new multiparticle modelisation of multilayered materials (M4)
}

\author{
Taeb Naciri, Alain Ehrlacher, Armelle Chabot
}

\section{To cite this version:}

Taeb Naciri, Alain Ehrlacher, Armelle Chabot. Interlaminar stress analysis with a new multiparticle modelisation of multilayered materials (M4). Composites Science and Technology, 1998, 58 (3-4), pp.337-343. 10.1016/S0266-3538(97)00085-7 . hal-01545526

\author{
HAL Id: hal-01545526 \\ https://hal.science/hal-01545526
}

Submitted on 22 Jun 2017

HAL is a multi-disciplinary open access archive for the deposit and dissemination of scientific research documents, whether they are published or not. The documents may come from teaching and research institutions in France or abroad, or from public or private research centers.
L'archive ouverte pluridisciplinaire HAL, est destinée au dépôt et à la diffusion de documents scientifiques de niveau recherche, publiés ou non, émanant des établissements d'enseignement et de recherche français ou étrangers, des laboratoires publics ou privés. 


\title{
Interlaminar stress analysis with a new multiparticle modelisation of multilayered materials (M4)
}

\author{
T. Naciri, A. Ehrlacher \& A. Chabot \\ Centre d'Enseignement et de Recherche en Analyse des Matériaux \\ Ecole Nationale des Ponts et Chaussées \\ 6 et 8 avenue Blaise Pascal - Cité Descartes -Champs sur marne \\ 77455 Marne La Vallée cedex 2
}

\begin{abstract}
We propose a new model to calculate the interlaminar stresses in a multilayered medium. It is well known that the classical analysis methods of multilayered materials do not give enough information about the interlaminar stresses that causes damages. The model we propose describe the multilayered medium as a surface with as many particles in each point of the surface as the number of layers in the medium. For this reason, it is named multiparticle modelisation of multilayered materials (M4 in the following of the text). It permits to calculate directly the stresses as interaction between particles at the same point of the surface. In a certain sense, the M4 model belongs to the same family than the well known Shear-lag model (Garett \& Bailey ${ }^{9}$ ) (Macquire \& al. ${ }^{10}$ ) and the Pagano's global-local model (Pagano \& al ${ }^{14}$ ). It is more general than the shear-lag's one and simpler than the Pagano's global-local model. We present in this paper the equations of the model and we establish analytical solution for a cylindrical bending multilayered plate problem. Through this example, we show that we can have a good prediction of displacements and shear stresses and that the M4 model is a pertinent tool to study interlaminar stresses.
\end{abstract}

Keywords : COMPOSITE MATERIALS - INTERLAMINAR STRESS

\section{INTRODUCTION}

Classical Plate Theories (Love-Kirchhoff, Reissner-Mindlin) do not allow to calculate the interlaminar stresses or the edge stresses (edge effects) in the multilayered composite materials. These stresses are important in the delamination of composite materials and other damaging processes. For this reason, several authors have been interested by the study of these interlaminar stresses and have proposed analytical solutions in particular cases (Pagano ${ }^{12}$ ), or numerical tridimensionnal models to process more general cases $\left(\right.$ Rybicki $\left.{ }^{16}\right)$ (Renieri \& Herakovich $\left.{ }^{15}\right)\left(\right.$ Wang \& Crossman $\left.^{19}\right)\left(\right.$ Spilker \& Chou $\left.{ }^{18}\right)$. These approaches have shown the concentration of the normal stress between two layers in the vicinity of edges with sometimes a weak singularity of the fields (Sanchez Palancia ${ }^{17}$ ). General tridimensional analysis with finite element method are difficult to use because they need a very thin mesh and a long time of calculation. 
Then, many authors have adopted simplified approaches aiming to make less calculations. A first family of these simplified approaches consists in using plate theory (that do not verify therefore $3 \mathrm{D}$ boundary conditions) and determining in the vicinity of the edges corrective stress and strain fields to balance the equilibrium error at the edge $\left(\right.$ Allix $\left.{ }^{l}\right)$ (Dumontet ${ }^{5}$ ). These methods are generally simple and predictive, but they need two consecutive calculations, so it is interesting to look for other simplified methods that solves the problem with a single analysis. From one end of such a family of models to the other, we find the very classical shear-lag's model (Garett \& Bailey ${ }^{9}$ ) and the different Pagano's simplified models ${ }^{13}$ and the global local Pagano's model ${ }^{14}$. These models have in common the fact that the multilayered medium is geometricaly described as a surface and that the fields of the different layers are distinguished. We can say then that they are multiparticle models in the sense that in each point of the surface describing the multilayered medium, they have as many particles as the number of layers. These particles are simple points in the case of the shear-lag model. In the case of the Pagano's models, they are more complex.

One of the main characteristic of such multiparticle models of multilayered materials is the number of generalized displacements or, what is the same, the number of equilibrium equations.

In the case of the shear-lag model, this number is $2_{n}$ (where $n$ is the number of layers): the two plane components of the mean in-surface displacement in each layer.

In the case of the Pagano's model, this number is $7 u$ : the 3 components of the mean displacement of each layer, the 2 components of the layer normal rotation and the first and the second momentum of the normal component of displacement through the thickness of each layer (the physical sense of these last two fields is not as direct as the five previous ones).

In this paper, we present an other multiparticle model of multilayered materials with $2 n+1$ generalized displacement fields and equilibrium equations. Due to the relatively low number of fields, our model is far more simpler than the Pagano's ones, but it is complex enough to take into account the bending of the multilayered medium that the shear-lag model doesn't take into account. The prediction of the $m 42 n+1$ model is discussed in comparison with the prediction of other models in the case of a simple problem of a multilayered plate bending.

\section{The $m 42 n+1$ model}

The Multiparticle Modelisation of Multilayered Materials (M4) has been developed to facilitate the study of damages such as delamination or transverse micro-craking (Ehrlacher $\left.\& a l^{67}\right)\left(\right.$ Caron $\left.^{3}\right)\left(\right.$ Foret $\left.^{8}\right)$. We present in this part the $m 42 n+1$ model hypothesis as well as its equations.

We consider the object (multilayered plate or shell) as a surface of $R^{3}$ (see figure 1). If $n$ is the number of layers, there are $n$ material particles in each point of the surface. 


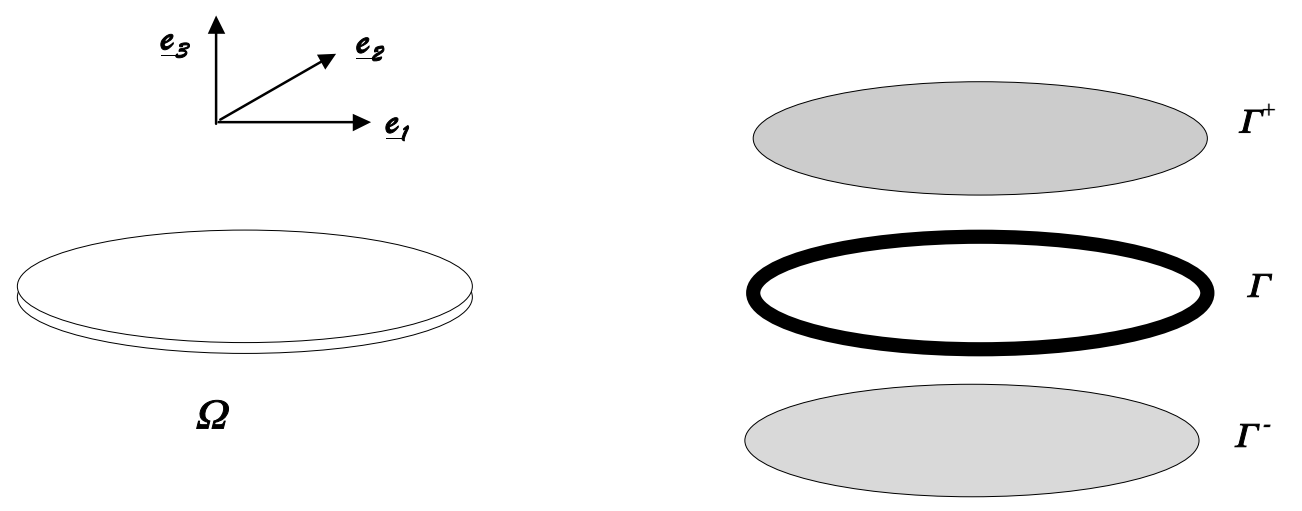

figure 1: The multilayered plate and its boundaries

The multilayered plate can be described as an open cylindrical domain $\Omega$ of $R^{3}$. Its base is $\omega \subset \mathbb{R}^{2}$. Let's note $\bar{h}_{i}^{-}, h_{i}^{+}$and $\bar{h}_{i}\left(\bar{h}_{i}=\frac{h_{i}^{-}+h_{i}^{+}}{2}\right)$ respectively the lower, the upper and the median height of the layer number $i\left(h_{i+1}^{-}=k_{i}^{+}\right)$and $e_{\iota}=h_{i}^{+}-h_{\imath}^{-}$its thickness.

$$
\Omega=\omega \times] h_{1}^{-}, h_{n}^{+}[
$$

let's note $\partial \omega$ the boundary of $\omega$. The boundary of $\Omega$ can be divided in three parts:

* The boundary of the plate

$$
\begin{aligned}
& \Gamma=\partial \omega \times] k_{1}^{-}, h_{n}^{+}[ \\
& \Gamma^{+}=\omega \times\left\{h_{n}^{+}\right\} \\
& \Gamma^{-}=\omega \times\left\{h_{1}^{-}\right\}
\end{aligned}
$$

* The upper face

* The lower face

The multilayered plate is submitted to the following external forces:

Volumic forces in $\Omega$ : $\underline{f}(\underline{x})$ where $\underline{x}=x \underline{e}_{1}+y \underline{e}_{2}+z \underline{e}_{3}$ is a point of $R^{3}$

Surfacic forces on $\Gamma$ :

$$
\underline{t}(\underline{x}) \quad \underline{x} \in \Gamma
$$

Surfacic forces on $\Gamma^{+}$:

$$
\underline{\tau}^{+}(\underline{x}) \quad \underline{x} \in \Gamma^{+}
$$

Surfacic forces on $\Gamma^{-}$:

$$
\underline{t}^{-}(\underline{x}) \quad \underline{x} \in \Gamma^{-}
$$

Let's develop the $m 42 n+1$ model by using the virtual power principle for the static case. The kinetic fields are given by $n$ velocity fields. We choose a virtual velocity field for which each layer of the laminate has its own in-surface motion, but with the same orthogonal velocity for all the layers:

$$
\left.\underline{V}^{*}(x, y, z)=\underline{V}^{i^{*}}(x, y)=V_{\alpha}^{i^{*}}(x, y) \underline{e}_{\alpha}+W^{*}(x, y) \underline{e}_{3} \quad \alpha=1,2 \quad \text { for } z \in\right] h_{i}^{-}, h_{i}^{+}[i=1, n
$$

The external virtual power $\mathcal{P}_{e}$ and the internal virtual power $\mathcal{P}_{i}$ can be written as follows: 


$$
\begin{aligned}
& P_{e}\left(\underline{v}^{*}\right)=\int_{\Omega} \underline{f}(x, y, z) \cdot \underline{v}^{*}(x, y, z) d \Omega+\int_{\Gamma} \underline{t}(x, y, z) \cdot \underline{v}^{*}(x, y, z) d \Gamma \\
&+\int_{\omega} \underline{t}^{+}\left(x, y, h_{u}^{+}\right) \cdot \underline{v}^{*}\left(x, y, h_{u}^{+}\right) d \omega+\int_{\omega} \underline{t}^{-}\left(x, y, h_{l}^{-}\right), \underline{v}^{*}\left(x, y, h_{l}^{-}\right) d \omega
\end{aligned}
$$

By noting $Z_{\alpha}^{i}(x, y)=\int_{a_{i}^{-}}^{k_{i}^{+}} \sigma_{\alpha}(x, y, z) d z \quad$ and $\quad \gamma_{\alpha}^{i}(x, y)=\int_{a_{i}^{-}}^{b_{i}^{+}} t_{\alpha}(x, y, z) d z \quad(i=1, n ; \alpha=1,2)$, and $z_{3}(x, y)=\int_{k_{1}^{-}}^{h_{3}^{+}} f_{3}(x, y, z) d z+t_{3}^{+}\left(x, y, h_{n}^{+}\right)+t_{3}^{-}\left(x, y, h_{1}^{-}\right)$and $z_{3}(x, y)=\int_{k_{1}^{-}}^{h_{3}^{+}} t_{3}(x, y, z) d z$ we have:

$$
\begin{aligned}
P_{c}\left(V^{*}\right)= & \left.\int_{\omega}\left(\sum_{i=1}^{n} z_{\alpha}^{i}(x, y), V_{\alpha}^{i^{*}}(x, y)\right) d \omega+\int_{\partial \omega}\left(\sum_{i=1}^{n} 7_{\alpha}^{i}(x, y), V_{\alpha}^{i^{*}}(x, y)\right)\right) d \gamma \\
& +\int_{\omega} z_{3}(x, y), w^{*}(x, y) d \omega+\int_{\partial \omega} 7_{3}(x, y), w^{*}(x, y) d \gamma \quad \alpha=1,2(3) \\
& +\int_{\omega} t_{\alpha}^{+}\left(x, y, h_{\alpha}^{+}\right), V_{\alpha}^{u^{*}}(x, y) d \omega+\int_{\omega} t_{\alpha}^{-}\left(x, y, h_{1}^{-}\right), V_{\alpha}^{i^{*}}(x, y) d \omega
\end{aligned}
$$

The internal virtual power can be written as follows:

$$
P_{i}\left(\underline{V}^{*}\right)=-\int_{\Omega} \sigma_{i q}(x, y, z), \varepsilon_{q y}\left(\underline{V}^{*}(x, y, z)\right) d \Omega \quad i, j=1,2,3
$$

The choice of the virtual velocity field leads to:

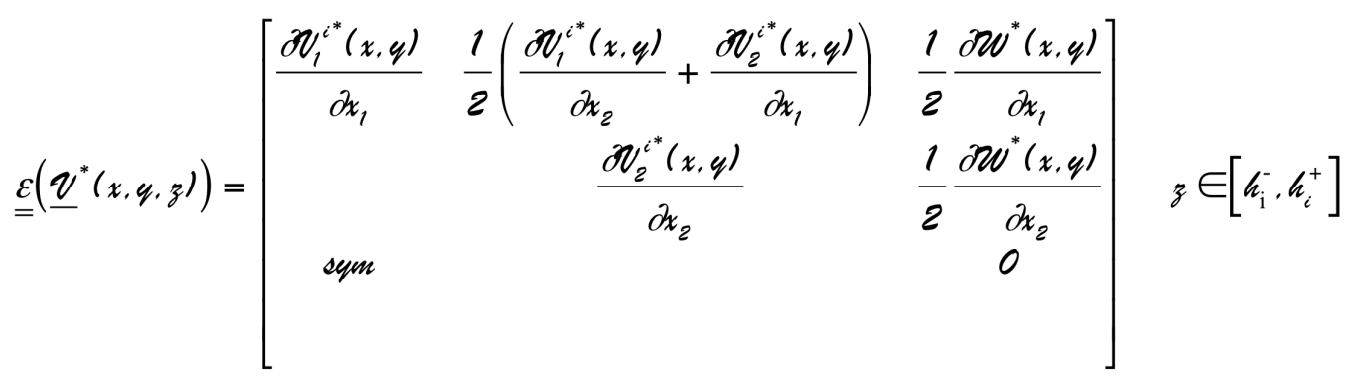

By taking into account the virtual in-surface velocity field discontinuities at the interfaces between layers and the virtual velocity field form, $\boldsymbol{P}_{i}$ becomes:

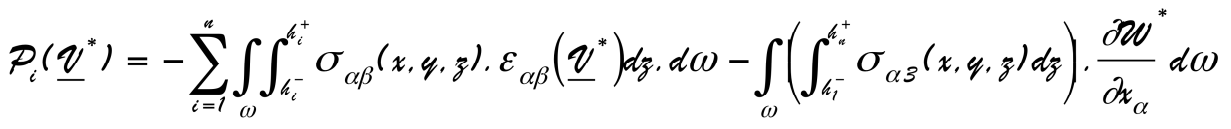

$$
\begin{aligned}
& -\sum_{i=1}^{n-1} \int_{\omega} \sigma_{\alpha 3}\left(x, y, k_{i}^{+}\right) \cdot\left(v_{\alpha}^{i+i^{*}}-V_{\alpha}^{i^{*}}\right) d \omega \quad \alpha, \beta=1,2
\end{aligned}
$$

In the expression of the virtual internal power, we recognize the following generalized stress fields:

$$
N_{\alpha \beta}^{i}(x, y)=\int_{k_{i}^{-}}^{k_{i}^{+}} \sigma_{\alpha \beta}(x, y, z) d z \quad \alpha, \beta=1,2
$$

the components of membranar stress tensor in the layer number $i$. 


$$
\mathbf{Q}_{\alpha}(x, y)=\int_{h_{1}^{-}}^{h^{+}} \sigma_{\alpha 3}(x, y, z) d z \quad \alpha=1,2
$$

the components of the vertical shear in the multilayered plate.

$$
\tau_{\alpha}^{i, c+1}(x, y)=\sigma_{\alpha 3}\left(x, y, h_{i}^{+}\right) \quad \alpha=1,2
$$

the interlaminar shear stresses at the interface number $i, i+1(i=1, n-1)$.

By introducing (7), (8) and (9) in (6) the internal virtual power becomes:

$$
\begin{aligned}
P_{i}\left(\underline{\mathcal{V}}^{*}\right)=-\sum_{i=1}^{n} \int_{\omega} N_{\alpha \beta}^{i} \cdot \frac{\partial \mathcal{O}_{\alpha}^{i^{*}}}{\partial x_{\beta}} d \omega & -\int_{\omega} Q_{\alpha} \cdot \frac{\partial W^{*}}{\partial x_{\alpha}} d \omega \\
& \left.-\sum_{i=1}^{u-1} \int_{\omega}^{i, i+1} \tau_{\alpha}^{i+V_{\alpha}^{*}}-V_{\alpha}^{i^{*}}\right) d \omega \quad(\alpha, \beta=1,2)
\end{aligned}
$$

The application of the virtual power principle in the static case leads to equilibrium equations (11) and boundary conditions (12):

$$
\left\{\begin{array}{l}
\frac{\partial N_{11}^{i}}{\partial x_{1}}+\frac{\partial N_{12}^{i}}{\partial x_{2}}+\tau_{1}^{i, i+1}-\tau_{1}^{i-1, i}+\xi_{1}^{i}=0 \\
\frac{\partial N_{12}^{i}}{\partial x_{1}}+\frac{\partial N_{22}^{i}}{\partial x_{2}}+\tau_{2}^{i, i+1}-\tau_{2}^{i-1, i}+\xi_{2}^{i}=0 \\
\frac{\partial Q_{1}}{\partial x_{1}}+\frac{\partial Q_{2}}{\partial x_{2}}+\xi_{3}=0
\end{array} \quad \forall(x, y) \in \omega \quad i=1, n\right.
$$

where $\tau_{\alpha}^{0.1}=-t_{\alpha}^{-}$and $\tau_{\alpha}^{n, n+1}=t_{\alpha}^{+}(\alpha=1,2)$.

$$
\left\{\begin{array}{l}
\mathbf{N}_{11}^{i} \cdot n_{1}+\mathbf{N}_{12}^{i} \cdot n_{2}=T_{1}^{i} \\
\mathbf{N}_{12}^{i} \cdot n_{1}+\mathbf{N}_{22}^{i} \cdot n_{2}=T_{2}^{i} \\
\mathbf{Q}_{1} \cdot n_{1}+\mathbf{Q}_{2} \cdot n_{2}=T_{3}
\end{array} \quad \forall(x, y) \in \partial \omega \quad i=1, n\right.
$$

where $\underline{n}=u_{1} \underline{e}_{1}+n_{2} \underline{e}_{2}$ is the normal vector at a point $(x, y)$ of the boundary $\partial \omega$.

We can see from the equilibrium equations that the two first equations are the same that in the Shear-lag analysis. Nevertheless, the equation related to the orthogonal direction doesn't exist in the Shear-lag model. We have then $2 n+1$ equilibrium equations in our model and the Shearlag has $2 n$.

In order to identify the generalized constitutive law, we need to imagine the form of the stress field components. We will then assume that the 3D stress field components can be approximated by polynomial functions of $z$. We can notice that because the layer thickness in multilayered plates is generally very small, the in-surface $3 \mathrm{D}$ stress field components $\sigma_{\alpha \beta}(\alpha, \beta=1,2)$ can be approximated by $\sigma_{\alpha \beta}^{\alpha \alpha \beta}(\alpha, \beta=1,2)$ that is constant in $z$ in each layer: 


$$
\sigma_{\alpha \beta}^{a p p}(x, y, z) \approx \frac{1}{e_{i}} \mathbf{N}_{\alpha \beta}^{i}(x, y) \quad z \in\left[h_{i}^{-}, h_{i}^{+}\right] \quad i=1, n \quad \alpha, \beta=1,2
$$

By using the 3D equilibrium equations, we find that the shear stress field components $\sigma_{\alpha 3}(\alpha=1,2)$ can be approximated by $\sigma_{\alpha 3}^{a, \beta}(\alpha, \beta=1,2)$ that is linear on $z$ in each layer. As $\tau_{\alpha}^{i-1, i}=\sigma_{\alpha 3}\left(h_{i}^{-}\right)$and $\tau_{\alpha}^{i, i+1}=\sigma_{\alpha 3}\left(h_{i}^{+}\right), \sigma_{\alpha 3}^{\alpha \beta \mu}$ is then a piecewise linear function of $g$ :

$$
\begin{array}{rlrl}
\sigma_{\alpha \xi}^{a, j}(x, y, z)= & \frac{1}{2}\left(\tau_{\alpha}^{i, i+1}(x, y)+\tau_{\alpha}^{i-1 . i}(x, y)\right) & \alpha=1,2 & \\
& +\frac{z-\bar{h}_{i}}{e_{i}}\left(\tau_{\alpha}^{i, i+1}(x, y)-\tau_{\alpha}^{i-1 . i}(x, y)\right) & z \in\left[h_{i}^{-}, h_{i}^{+}\right] \quad i=1, n-1
\end{array}
$$

The relation (14) leads to the next form for the vertical shear in the multilayered plate (8):

$$
Q_{\alpha}(x, y)=\sum_{i=1}^{n}\left(\frac{\tau_{\alpha}^{i, i+1}(x, y)+\tau_{\alpha}^{i-1, i}(x, y)}{2}\right) \cdot e_{i}
$$

The component $\sigma_{33}$ of the stress tensor can therefore be aproximated with the help of the third 3D equilibrium equation by a parabolic function of $z$ in each layer. We are not interested in what follows by the expression of $\sigma_{33}$ because it does not appear in the internal virtual power. Furthermore, its contribution to the elastic energy will be neglected.

The generalized strain appears as the associated factors of the generalized stresses in the internal virtual power when the virtual velocity field is taken equal to the displacement field. If the displacement field is:

$$
\underline{u}(x, y, z)==u_{\alpha}(x, y, z) \underline{e}_{\alpha}+w(x, y, z) \underline{e}_{3} \quad \alpha=1,2
$$

and if we note the average components of the displacement field:

$$
\left\{\begin{array}{l}
u_{\alpha}^{i}(x, y)=\frac{1}{e_{i}} \int_{b_{i}^{-}}^{b_{i}^{+}} u(x, y, z) d z \quad \alpha=1,2 ; i=1, n \\
W(x, y)=\frac{1}{k_{a}^{+}-h_{1}^{-}} \int_{k_{i}^{-}}^{k_{a}^{+}} u(x, y, z) d z
\end{array}\right.
$$

The internal power for this displacement field is:

$$
\begin{aligned}
P_{i}(\underline{u})=-\sum_{i=1}^{n} \int_{\omega} N_{\alpha \beta}^{i} \frac{\partial \mathcal{u}_{\alpha}^{i}}{\partial x_{\beta}} d \omega & -\int_{\omega} \sum_{i=1}^{n} e_{i}\left(\frac{\tau_{\alpha}^{i, i+1}(x, y)+\tau_{\alpha}^{i-1, i}(x, y)}{2}\right), \frac{\partial U}{\partial x_{\alpha}} d \omega \\
& -\sum_{i=1}^{n-1} \int_{\omega} \tau_{\alpha}^{i, i+1}\left(\boldsymbol{u}_{\alpha}^{i+1}-\boldsymbol{U}_{\alpha}^{i}\right) d \omega \quad \alpha, \beta=1,2
\end{aligned}
$$

We can identify the following generalized strain associated to the membranar stress tensor in each layer: 


$$
\varepsilon_{\alpha \beta}^{i}=\frac{1}{2}\left(\frac{\partial \mathcal{U}_{\alpha}^{i}}{\partial x_{\beta}}+\frac{\partial \mathfrak{U}_{\beta}^{i}}{\partial x_{\alpha}}\right) \quad i=1, n \alpha, \beta=1,2
$$

that we call the in-surface deformation tensor in each layer. We can also identify the generalized strain associated to the interlaminar shear stress at each interface:

$$
\mathcal{D}_{\alpha}^{i, i+1}=U_{\alpha}^{i+1}-U_{\alpha}^{i}+\frac{e_{i}+e_{i+1}}{2}, \frac{\partial W}{\partial x_{\alpha}} \quad i=1, n-1 \quad \alpha=1,2
$$

that we call the shear deformation at each interface between two layers.

We can notice that in the Shear-lag analysis, the shear deformation is only related to the difference of the in-surface displacements and not to the derived expressions of the orthogonal displacement.

The identification of the generalized constitutive law is then classical. Let's write the elastic energy associated to the real stress field $\underline{\underline{\sigma}}$.

$$
\bar{W}(30)=\sum_{i=1}^{n} \int_{k_{i}^{-}}^{k_{i}^{+}}\left[\int_{\omega} \frac{1}{2} \sigma_{k t} S_{l u \omega}^{i} \sigma_{\Delta r} d \omega\right] d z \quad k, \ell, r, d=1,2,3
$$

where $S_{\text {cena }}^{i}$ are components of the compliance tensor of the layer number $i$. Each layer being orthotropic, this expression can be written as (if we assume that the axis 3 is an orthotropic axis):

$$
\begin{aligned}
\bar{w}(3 D)=\frac{1}{2} \sum_{i=1}^{n} \int_{k_{i}^{i}}^{k_{i}^{+}} & {\left[\int _ { \omega } \left(\sigma_{\beta \alpha} S_{\alpha \beta \gamma \delta}^{i} \sigma_{\partial \gamma}+2 \sigma_{\beta \alpha} S_{\alpha \beta 33}^{i} \sigma_{33}\right.\right.} \\
& \left.\left.+\sigma_{33} S_{3333}^{i} \sigma_{33}+4 \sigma_{3 \alpha} S_{\alpha 3 \beta 3}^{i} \sigma_{3 \beta}\right) d \omega\right] \mathrm{d} z \\
& \alpha, \beta, \gamma, \delta=1,2
\end{aligned}
$$

Let's assume that the contribution of $\sigma_{33}$ to elastic energy is negligible. After replacing $\underline{\underline{\sigma}}$ by its approximation in function of the components of the generalized stress fields (equation 18 $\& 19)$ of the $M a+n+1$ model, the approximate elastic energy can be written as:

$$
\begin{array}{r}
\bar{w}(30) \approx \bar{w}(2 n+1)=\int_{\omega}\left[\frac{1}{2} \sum_{i=1}^{n} \frac{1}{e_{i}} N_{\beta \alpha}^{i} S_{\alpha \beta \gamma \delta}^{i} N_{\delta \gamma}^{i}+\frac{2 e_{i}}{3} S_{\alpha 3 \beta 3}^{i}\left[2 \tau_{\alpha}^{i, i+1} \tau_{\beta}^{i, i+1}\right.\right. \\
\left.\left.+2 \tau_{\alpha}^{i-1 . i} \tau_{\beta}^{i-1 . i}+\tau_{\alpha}^{i-1 . i} \tau_{\beta}^{i, i+1}+\tau_{\alpha}^{i, i+1} \tau_{\beta}^{i-1 . i}\right]\right] d \omega \\
\alpha, \beta, \gamma, \delta=1,2
\end{array}
$$

The approximate constitutive law of the $m a 2 n+1$ model is finally written:

$$
\varepsilon_{\alpha \beta}^{c}=\frac{1}{e_{i}} S_{\beta \alpha \delta \gamma}^{c} N_{\gamma \delta}^{\iota}
$$




$$
\begin{aligned}
D_{\alpha}^{i, i+1}= & \frac{4}{3}\left[e_{i} S_{\alpha 3 \beta 3}^{i}+e_{i+1} S_{\alpha 3 \beta 3}^{i+1}\right] \tau_{\beta}^{i, i+1} \\
& +\frac{2}{3} e_{i+1} S_{\alpha 3 \beta 3}^{i+1}, \tau_{\beta}^{i+1, i+2}+\frac{2}{3} e_{i} S_{\alpha 3 \beta 3}^{i}, \tau_{\beta}^{i-1, i}
\end{aligned}
$$

We have put in obviousness in this model a coupling between the different interfaces in the compliance matrix of the $m 42 n+1$ model. That makes an other difference with the shear-lag analysis that has no coupling between interfaces.

Finally, we've just built a model for which the generalized stresses are the membranar stress tensor for each layer (7) and the interlaminar shear stress vector for each interface (9). These generalized stresses have to verify equilibrium equation (11) and boundary conditions (12). The associated generalized strains are the in-surface deformation tensor in each layer (19) and the shear deformation vector at each interface between layers (20). The approximate constitutive law is given by equations (24) and (25).

We can notice that we have choosen a virtual motion (1) that do not take into account the flexure of each layer. The model we build will of course take into account the flexure of the whole multilayered plate

With the $m a 2_{n}+1$ model we have just presented, it is possible to establish analytical solutions for simple cases which can be used to validate the $m 42 n+1$ model. The example we present consists in comparing for a multilayered medium constituted of a $\left(0^{\circ}, 90^{\circ}, 0^{\circ}\right)$ laminate, the deflexion and the shear stresses obtained by the $m a 2 n+1$ model and by classical multilayered plate theories.

\section{EXAMPLE : CYLINDRICAL BENDING}

In this example, we compare the deflection and the shear stresses obtained for a multilayered plate submitted to cylindrical bending by the $m a 2 n+1$ model, by the Love-Kirchhoff and the Reissner multilayered plates models (Berthelot ${ }^{2}$ ) and by the exact solution of Pagano ${ }^{11}$. We choose for that a multilayered plate constituted of a $\left(0^{\circ}, 90^{\circ}, 0^{\circ}\right)$ laminate infinitly width and loaded by a pression $p\left(x_{1}, x_{2}\right)=g_{0} \sin \left(\frac{\pi x_{1}}{a}\right)$ applied on its upper face. The Figure number 2 presents the plate geometry and the table 1 the constitutive material used by these authors.

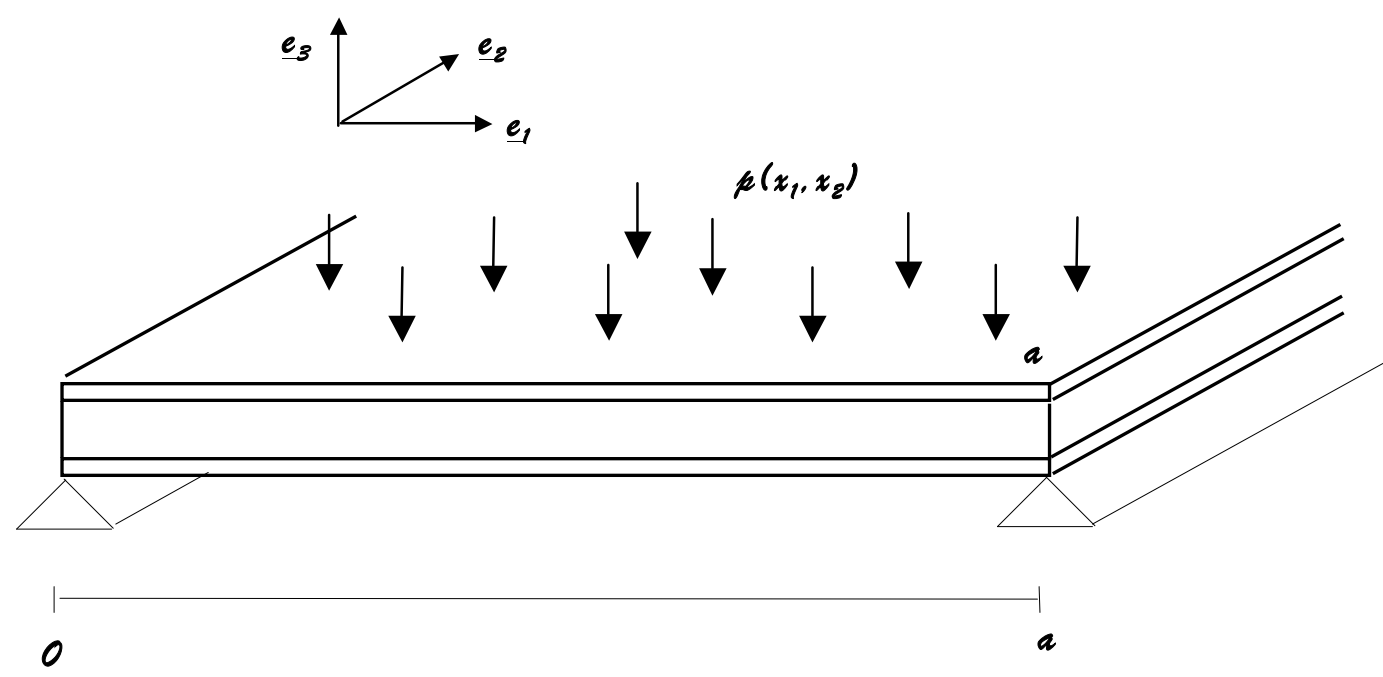


Figure 2: Plate geometry

\begin{tabular}{ccccc}
\hline table 1: The constitutive material & & & \\
\hline $\mathrm{E}_{1}$ & $\mathrm{E}_{2}=\mathrm{E}_{3}$ & $v_{12}=v_{23}$ & $\mathrm{G}_{12}=\mathrm{G}_{13}$ & $\mathrm{G}_{23}$ \\
\hline $2510^{6} \mathrm{psi}$ & $10^{6} \mathrm{psi}$ & 0.25 & $0.510^{6} \mathrm{psi}$ & $0.210^{6} \mathrm{psi}$ \\
\hline
\end{tabular}

We number layers from 1 to 3 from the bottom to the top. Let's note $k / 4$ the thickness of the layer 1 and 3 and $k / 2$ the thickness of the layer 2 . According to the plate geometry and to the loading symetries, the displacement fields (17) can be simplified to:

$$
U_{1}^{i}\left(x_{1}, x_{2}\right)=U_{1}^{i}\left(x_{1}\right) ; U_{2}^{i}\left(x_{1}, x_{2}\right)=U_{2}^{i}\left(x_{1}\right) ; W_{\left(x_{1}, x_{2}\right)=W\left(x_{1}\right)} \quad i=1,2,3
$$

By using the equilibrium equations (11), boundary conditions (12), the generalized constitutive law (24), (25) and the form of displacement (26), we obtain, after solving a differential system the maximum deflexion and the maximum interlaminar shear stresses:

$$
\begin{aligned}
& w_{2 a+1}=w(a / 2, y)=-\frac{128 q_{0} a^{4}}{9 \cdot \pi^{4} Q_{11} h^{3}}\left(1+\frac{\pi^{2}}{48} \frac{Q_{11}\left(G_{23}+3 G_{13}\right)}{G_{23} G_{13}}\left(\frac{h}{a}\right)^{2}\right) \\
& \tau_{i}^{1.2}(0, y)=\tau_{1}^{2.3}(0, y)=\frac{4 q_{0}}{3 \pi}\left(\frac{a}{h}\right)
\end{aligned}
$$

where $Q_{11}=\frac{E_{1}}{1-v_{12} v_{21}}$ and $G_{13}$ and $G_{23}$ are the shear modulus in the $\left(x_{1} x_{2}\right)$ plan.

The maximum deflexion obtained with the $m a 2 n+1$ model can be compared to the maximum deflexion obtained by several classical plate theories:

* The Love-Kirchhoff plate multilayered theory (Berthelot ${ }^{2}$ ) leads to the following maximum deflexion:

$$
w_{L x}=\frac{96 a^{4} q_{0}}{\pi^{4}\left(7+\frac{E_{2}}{E_{1}}\right) Q_{11} h^{3}}
$$

The Reissner plate multilayered theory (Berthelot ${ }^{2}$ ) leads to the following maximum deflexion:

$$
w_{R . k}=w_{L K}\left[1+\frac{\pi^{2}}{48 k} \frac{7+\frac{E_{2}}{E_{1}}}{1-v_{12} v_{21}} \frac{E_{1}}{G_{13}+G_{23}}\left(\frac{h}{a}\right)^{2}\right]
$$


where 6 is a corrective factor introduced in the transverse behaviour relationship to take into account in different ways some effects of the vertical shear in multilayered plates. In the literature $\left(\right.$ Berthelot $\left.^{2}\right)$ we often find these different values of $k-k=1,2 / 3$ and 5/6-.

The Pagano's exact solution in cylindrical bending ${ }^{11}$.

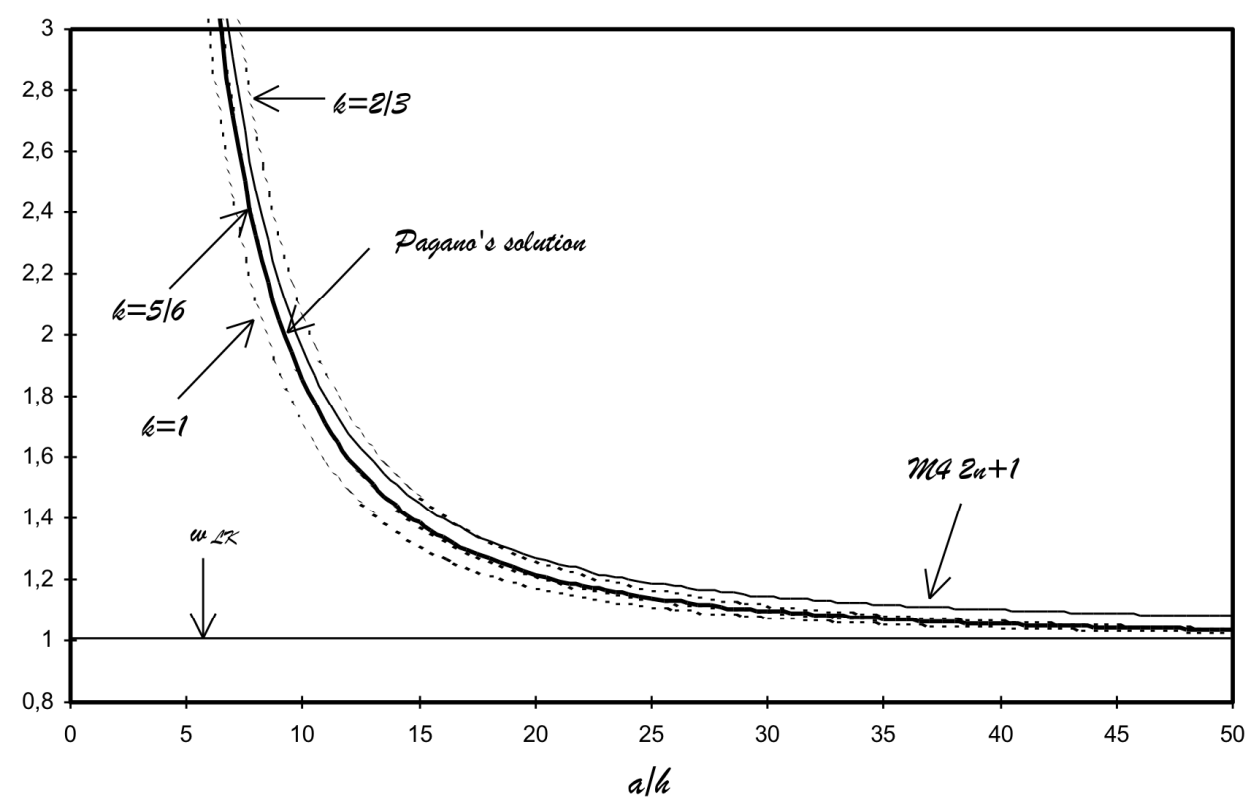

figure 3 : Evolution of the maximum deflexion for different theories

The figure 3 presents all these maximum deflexions for different $\left(\frac{a}{b}\right)$ ratios. These curves show that:

- The maximum deflexions obtained with the Reissner multilayered plates model with $k=5 / 6$ and Pagano's analytical solution are pratically equal.

- The $M 42 n+1$ model gives a very good approximation of the maximum deflexion for small $\left(\frac{a}{b}\right)$ ratios.

- For large $\left(\frac{a}{h}\right)$ ratios, the maximum difference between the $m a 2 n+1$ model and Pagano's analytical solution is $4 \%$. This result was expected because the $m 42 n+1$ model does not take into account the bending energy of each layer. The bending of the multilayered plate is taken into account through the interlaminar shear stress. If we increase the number of plies, the difference between the predictions of the $m a 2_{n}+1$ model and Pagano's analytical solution will diminish.

Furthermore, the interlaminar shear stresses are obtained directly and we can also compare the evolution of the stress $\left(\sigma_{13}\right)$ in the thickness of the laminate. The figures number 4 and 5 present the shear stress $\sigma_{13}$ calculated with the $m 42 n+1$ model and with the Pagano's exact solution at the edge $\left(x_{1}=0\right)$ for two different $\left(\frac{a}{b}\right)$ ratios $\left(\left(\frac{a}{b}\right)=4\right.$ and 10$)$. 


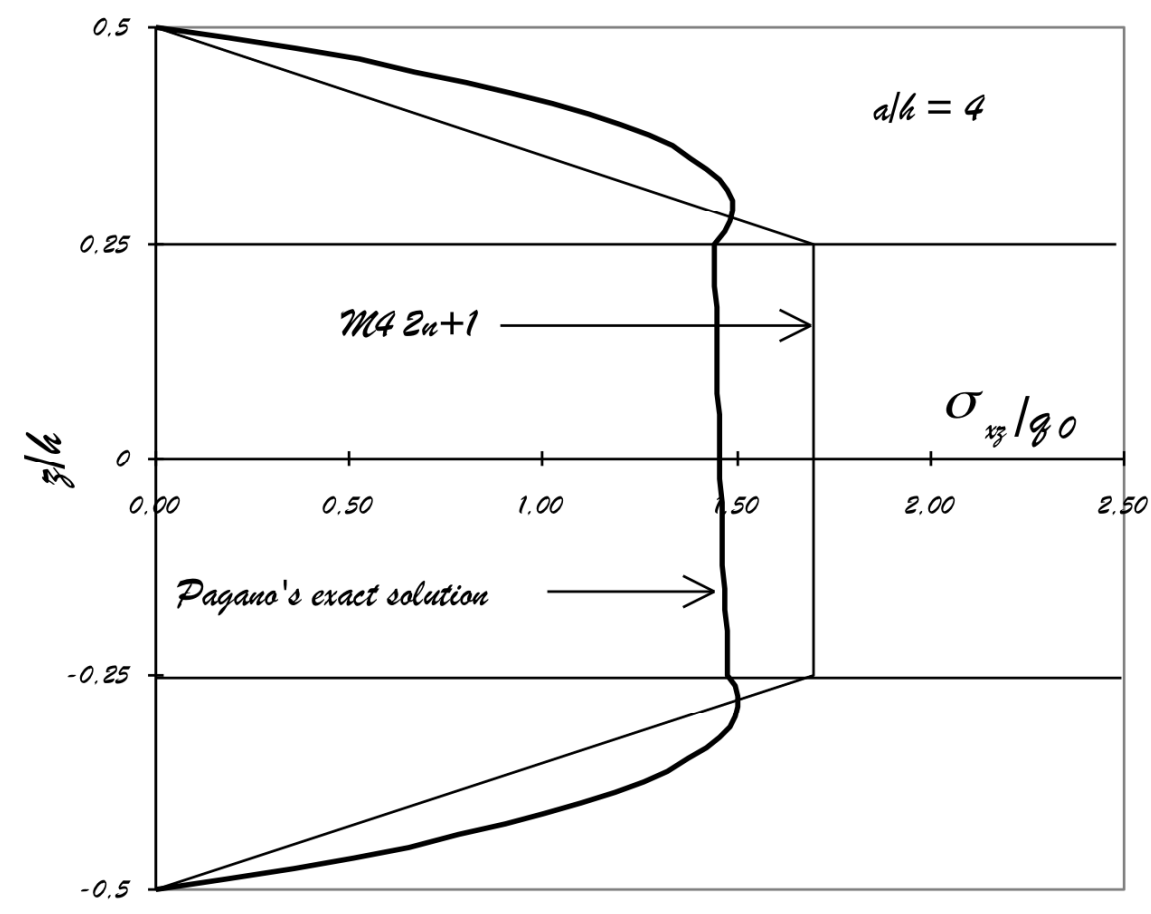

figure 4 : Evolution of the shear stress $\sigma_{13}$ in the thickness at the edge $\left(x_{1}=0\right)\left(\frac{a}{b}=4\right)$.

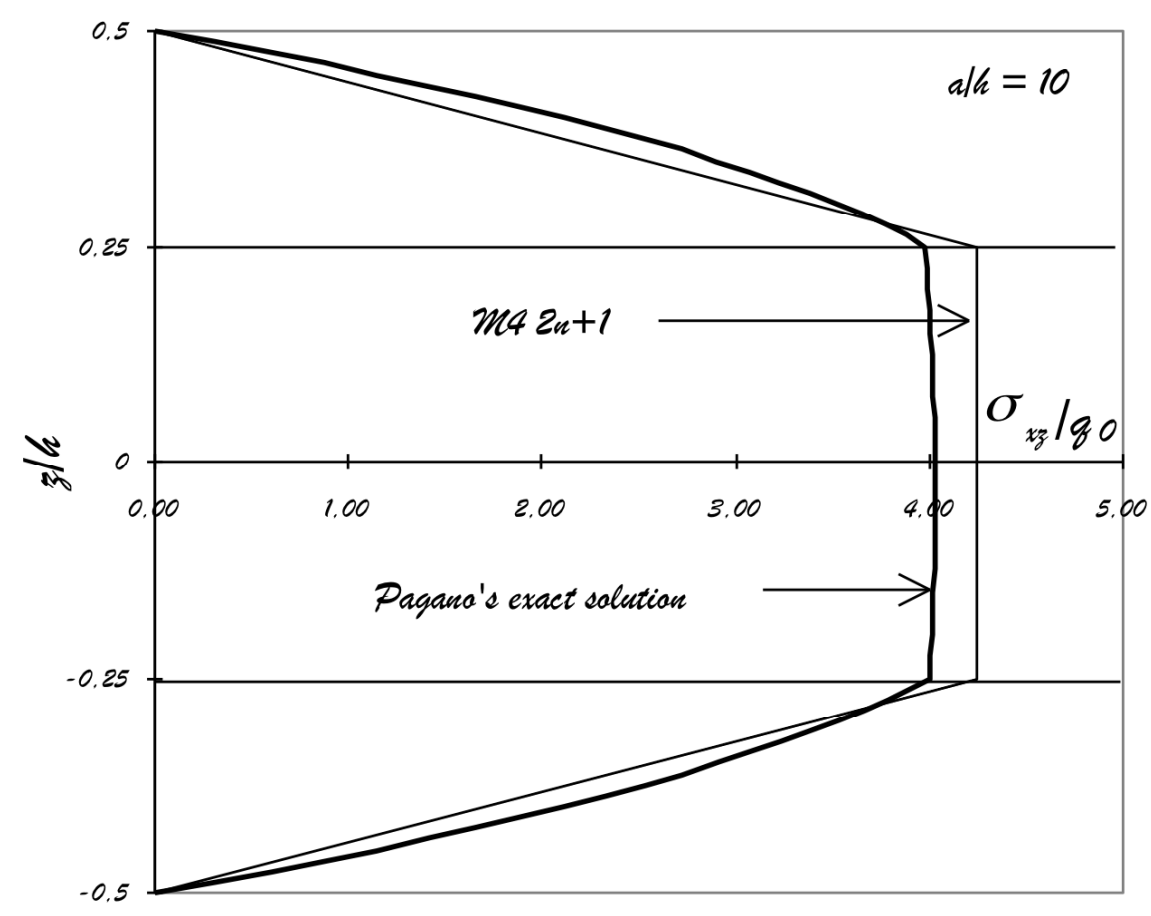

figure 5 : Evolution of the shear stress $\sigma_{13}$ in the thickness at the edge $\left(x_{1}=0\right)\left(\frac{a}{b}=10\right)$. 
We can notice that the $m 42 n+1$ model gives a good prediction of the shear stress evolution in the thickness even for thick multilayered plates (i.e. when the $\left(\frac{a}{h}\right)$ ratio is small). We remarque also that the approximate maximum shear stress calculated with the $m \boldsymbol{4} 2 n+1$ model is greater than the exact shear stress determined by Pagano. The $m 42 n+1$ model prediction is in the security side because the model overestimates the interlaminar shear stress.

\section{CONCLUSION}

We have proposed in this paper a new model to calculate the interlaminar stresses in a multilayered medium, the $m a 2 n+1$ model. It is more general than the shear-lag's (Garett \& Bailey ${ }^{9}$ ) and simpler than the Pagano's global-local model (Pagano \& al ${ }^{14}$ ). We have presented the equations of the model and established an analytical solution.

In the presented example, we have shown that the $m 42 n+1$ model gives good prediction of the maximum deflexion and the shear stress for a cylindrical bending multilayered plate problem. The case of a $\left(0^{\circ}, 90^{\circ}, 0^{\circ}\right)$ laminate was studied.

Otherwise, it is possible to build other models with an increasing number of generalized displacements. In $\left(\mathrm{Chabot}^{4}\right)$, many models are developped showing that while we increase to number of kinematic fields, we obtain a better description of the interlaminar stresses. In this family of models, the $m a 2 n+1$ model is a pertinent tool because we can have a quick and good estimation of interlaminar shear stresses.

\section{REFERENCES}

1. Allix O, Modélisation du comportement des composites stratifiés, Thèse de Doctorat de l'Université Pierre et Marie Curie, 1989.

2. Berthelot JM., Matériaux composites, Ed Masson, 1992.

3. Caron J.F., Fissuration tranverse des matériaux composites, thèse de l'Ecole Nationale des Ponts et Chaussées, 1993.

4. Chabot A., Analyse des efforts d'interface entre les couches des matériaux composites à l'aide de Modèles Multiparticulaires des Matériaux Multicouches (M4), thèse de l'ENPC, 1997.

5. Dumontet H, Homogénéisation et effets de bords dans les matériaux composites, Thèse d'Etat de l'Université Pierre et Marie Curie, 1990.

6. Ehrlacher A., Chabot A. \& Naciri T, Prévision de l'initiation du délaminage, Rapport de fin de contrat SNECMA n ${ }^{\circ} 486$ (CERAM ENPC), 1994.

7. Ehrlacher A., Naciri T., Chabot A. \& Caron J.F., Analyse des efforts d'interface à l'aide de la Modélisation Multiphasique des Matériaux Multicouches (M4), 9ème Journées Nationales sur les Composites (JNC9), SAINT-ETIENNE, 22/24 Novembre 1994

8. Foret G, Effets d'echelle dans la rupture des composites unidirectionnels, thèse de l'Ecole Nationale des Ponts et Chaussées, 1995.

9. Garett K.W., Bailey J.E., Multiple transverse fracture in $90^{\circ}$ cross-ply laminates of a glass fibre-reinforced polyster, J. Mat. Scien., Vol. 12, 1977.

10. Macquire B., Petitpas E., Valentin D., Experimental and theoretical damage accumulation in glass-epoxy tubes under torsion loading, Composites Polymers, Vol. 5, N³, 1992. 
11. Pagano N.J., Exact solutions composites laminates in cylindrical bending, J. Composite Mat., Vol. 3, 398-411, 1969.

12. Pagano N.J., Exact solutions for rectangular bidirectional composites and sandwich plates, J. Composite Mat., Vol. 4, 20-34, 1970.

13. Pagano NJ., Stress Fields in composite laminates, Int. J. Solids \& Struct., Vol. 14, 385400, 1978.

14. Pagano N.J., Soni S.R., Global-local variationnal model, Int. J. Solids Structures. Vol. 19, n³, 207-228, 1983.

15. Renieri G.D., Herakovich C.T., Nonlinear analysis of laminated fibrous composites, VPIE-76-10, Virginia Polytechnic Institute and State University, 1976.

16 Rybicki E.F., Approximate three-dimensional solutions for symmetric laminates under inplane loading, J. Composite Materials, Vol. 5, 1971.

17. Sanchez-Palancia E., Problèmes mathématiques de la mécanique. Influence de l'anisotropie sur l'apparition de singularités de bord dans les problèmes aux limites relatifs aux matériaux composites, C. R. Acad. Sc., t. 300, Série I, n¹, 1985.

18. Spilker R.L., Chou S.C., Some new results on edge effect in symmetric composite laminates, J. of Composite Materials, vol. $\mathrm{n}^{\circ} 11,92-106,1980$.

19. Wang A.S.D., Crossmann F.W., Some new results on edge effect in symmetric composite laminates, J. Composite Materials, Vol. 11, 1977. 